\title{
Design of Neuro-Fuzzy Controller for a Rotary Dryer
}

\author{
F. F. G. Areed \\ Professor of Computer \& systems \\ Eng. Dept. \\ Faculty of engineering \\ Mansoura university
}

\author{
M. S. El-Kasassy \\ Lecturer at Computer \& systems \\ Eng. Dept. \\ Faculty of engineering \\ Mansoura university
}

\author{
Kh. A. Mahmoud \\ Computer \& systems Engineer, \\ at Masria-Card Co. Cairo, Egypt
}

\begin{abstract}
Drying is a very complex non-linear process including the movement of solids in addition to the thermal drying. This means that both the modeling and control of a rotary dryer is difficult with conventional methods, and most of previous researches goes to directly linearize the non-linear model, estimating many parameters that affect the model or work on specific linear model so applying modern controllers even fuzzy or Neuro-Fuzzy always gives approximate results and valid only in certain operating points, in this paper we try to make a combination between two dynamic models of dryer plant to solve the drying rate equation which mainly causes the non-linearity of the model.
\end{abstract}

This article will validate the proposed model then applying modern adaptive control techniques Fuzzy and Neuro-Fuzzy controllers, comparing the behavior of the plant dryer when connected to each of this controllers versus the conventional PID, the aim is to improve dryer control, this will lead to energy, cost savings and increasing efficiency of the drying, the behavior of the control systems has been tested with simulations based on the model of a plant dryer using The Matlab®, and Simulink and Fuzzy Logic Toolbox, ANFIS Toolbox.

\section{Key Words}

Rotary Dryer, Fuzzy control, Neuro-Fuzzy control

\section{INTRODUCTION}

Drying, especially rotary drying is without doubt one of the oldest and most common unit operation in industry, in this paper, we will focus on the process of drying solids (aggregates) as a part of manufacturing the Hot Mix Asphalt used in roads construction.

Rotary drying is an operation which is easy and reliable, but neither energy-efficient nor environmentally friendly. Most rotary dryers, especially older ones, are still controlled partially manual, relying on the human "eye" and experience of the operator,

Drying is an operation of great commercial importance, in all industrial applications ranging through the food, agricultural, mining and manufacturing sectors.

Modern society requires better product quality, improved safety practices and more environmentally benign operations, as well as higher productivity, better energy efficiency and reduced material wastage. As drying is certainly one of the most energyintensive operations in industry, and as most dryers operate at low thermal efficiency, the development of models and control systems to improve dryer operation and efficiency [8].

\subsection{ROTARY DRYING}

Large quantities of granular material with particles of $10 \mathrm{~mm}$ or larger that are not too fragile or heat sensitive or cause any other solids handling problems are dried in rotary dryers in the process industries. The rotary dryer is one of the most common types of industrial dryer. It is a cylindrical shell usually constructed from steel plates, slightly inclined,

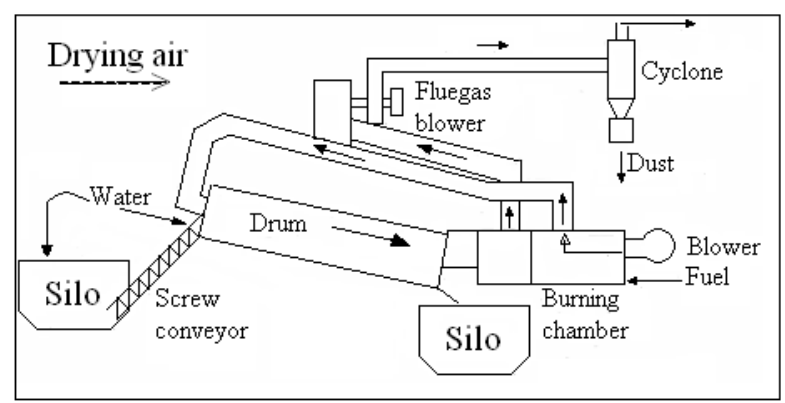

Fig 1: Structure of rotary dryer plant

An array of lifting flights of various shapes is constructed inside the shell to shower the solids in order to ensure contact with the gas.

These flight configurations vary from spirals to straight flights. The effect of the flight design i.e. the number of flights, their dimensions and their shape, on the performance of the dryer is very complicated.

A rotary dryer has two distinct functions: as a conveyor and as a heating device. The movement of solids through the dryer is influenced by the following mechanisms: lifting, cascade action, sliding and bouncing, as depicted in Figure.

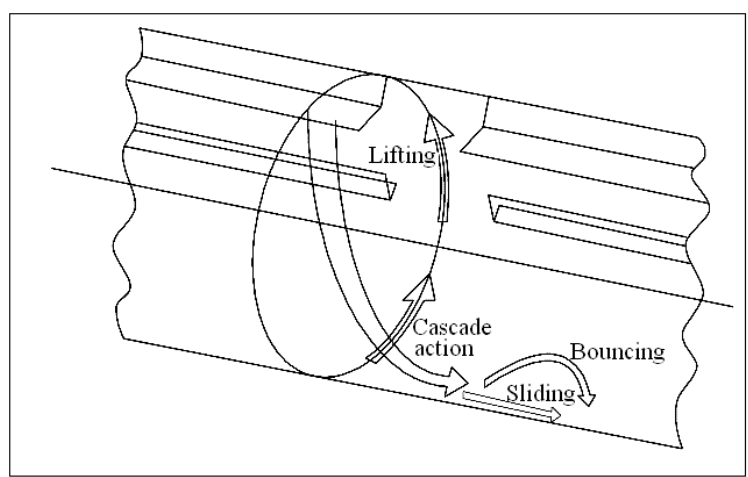

Fig 2: A cascading rotary dryer (Baker 1983) 
As the dryer rotates, solids are picked up by the flights, lifted for a certain distance around the drum and showered through the air in a cascading curtain. Most of the drying occurs at this time, as the solids are in close contact with the gas. Flight action is also partly responsible for the transport of solids through the drum.

Drum is typically $0.3-5 \mathrm{~m}$ in diameter, $5-90 \mathrm{~m}$ in length and rotating at $1-5$. It is usually operated with a negative internal pressure to prevent dust escape. Solids introduced at the upper end move towards the lower or discharge end. Depending on the arrangement for the contact between the drying gas and the solids, a dryer may be classified as direct or indirect, concurrent or counter-current [4].

The factors affecting the modeling of a rotary dryer can be classified as follows (Kelly 1995):

- $\quad$ physical properties of the solids, such as particle size and shape, density and moisture content,

- dryer variables, such as the diameter and length of the drum and the design and number of lifting flights and

- Operating conditions, such as the feed flow and temperature, the flow and temperature of the drying air and the slope and rotation speed of the drum.

All the above factors affect the heat transfer in the drum, and all except the temperatures of the solids and drying air have an effect on the loading (hold up) and time of passage of the drum [13]. The hold up of solids has a great effect on the operation of the dryer, as an excessively low hold up will reduce the production rate but too great a hold up will make material roll on the bottom of the dryer so that the desired moisture content will not be achieved and the power required to turn the dryer will be increased.

A hold up of 3-15\% of the total drum volume, with values in the range 8-12\% most common, gives satisfactory operation (Keey 1995). The thermal efficiency of a rotary dryer depends on the manner of drying and varies over a wide range from 25 $\%$ in an indirect-fired system to $85 \%$ in a steam tube (McKetta \& Cunningham 1983).

Although rotary dryers have been used for many decades in numerous industrial sectors, research into their modeling and control has been limited and can be said to be still in its infancy.

\subsection{The basic objectives for the development of dryer control are:}

- Maintain the desired product moisture content in spite of disturbances in drying operation,

- Maximize production with optimal energy use and at minimal costs so that the costs of investment in automation are reasonable compared with other equipment costs,

- Avoid over drying, which increases energy costs and can cause thermal damage to heat-sensitive solids, and

- $\quad$ Stabilize the process.

\section{MODEL OF THE PLANT DRYER 2.1 Dynamic models for the plant dryer:}

A rotary dryer is a distributed parameter system in which both temperature and moisture are functions of time and distance, according to the general equation: $f_{i}\left(x_{i}, 1, \mathrm{t}\right)=\frac{\partial x_{i}(1, \mathrm{t})}{\partial \mathrm{t}} \pm v_{i}(t) \frac{\partial x_{i}(1, \mathrm{t})}{\partial \mathrm{l}}$

Where:

$\mathrm{Xi}$ : moisture or temperature in the solids or gas phase

$\mathrm{Vi}$ : linear velocity in the solids or gas phase

1: axial co-ordinate, $\mathrm{t}$ : time.

A positive sign for vi applies to con-current drying and a negative sign to counter-current drying.

The distributed parameter model is complex and cumbersome to handle, and the temperature and especially the content of solids and drying air inside the dryer are difficult to measure. It is therefore simplified to a lumped parameter model in which the partial derivative of the axial co-ordinate length equals to the total length of the drum.

The equation for the gas moisture content is not included in the overall model for the dryer, because it is not measured in the pilot dryer. The model is now of form:

$$
\begin{aligned}
& \frac{d \mathrm{X}_{s, \text { out }}}{d t}+V_{s} \frac{\left(\mathrm{X}_{s, \text { out }}-\mathrm{X}_{s, \text { in }}\right)}{\mathrm{L}}=-R_{w} \\
& C_{s} \frac{d \mathrm{~T}_{s, \text { out }}}{d t}+V_{s} C_{s} \frac{\left(\mathrm{T}_{s, \text { out }}-\mathrm{T}_{s, \text { in }}\right)}{\mathrm{L}}= \\
& -\frac{\mathrm{U}_{v} \mathrm{~V}_{v}}{\mathrm{~F}_{s}}\left(\mathrm{~T}_{g, \text { out }}-\mathrm{T}_{s, \text { in }}\right)-\lambda R_{w} \\
& C_{g} \frac{d \mathrm{~T}_{g, \text { out }}}{d t}+V_{g} C_{g} \frac{\left(\mathrm{T}_{g, \text { out }}-\mathrm{T}_{g, \text { in }}\right)}{\mathrm{L}}= \\
& -\frac{\mathrm{U}_{v} \mathrm{~V}_{v}}{\mathrm{~F}_{g}}\left(\mathrm{~T}_{g, \text { out }}-\mathrm{T}_{s, \text { out }}\right)-\lambda \frac{F_{s}}{F_{g}} R_{w}
\end{aligned}
$$

where the meaning of symbols are, as in Yliniemi (1999):[4] $\mathrm{Xs}$, out, Tg,out and Ts,out are the moisture content, the temperature of gas and of solids in the output.

The model is non-linear, because the drying rate $\mathrm{Rw}$, which describes the course of drying inside the solids, is generally a non-linear function of solids characteristics and drying air temperature in the falling rate period, as many researchers have found in their experimental [1], [2], [5], some researchers have introduced mono-variable models and multi-variable models to simulate the dryer [3], [10].

\subsection{Drying rate equation:}

According to the equations above, we can notice that Rw is the drying rate of the product. This is one of the most important parameters of the model and it must be experimentally determined.

According to Baker (1983), it should include moisture solid equilibrium data and experimentally determined batch drying curve obtained under conditions approximating as closely as possible those of the process that it is being modeled. For rotary dryers, the batch equipment that Nonhebel and Moss (1979) recommended if the product does not a form hard scab when drying, are direct dryers with cross-flow of air or small rotary dryers.

In this study a thin layer dryer has been used This kind of equipment has been used by authors like Papadakis et al. (1994) and Cao and Langrish (2000) to study the drying kinetic and the expressions obtained have been used to model the rotary drying process. Drying experiments of a mixture of product similar to that obtained in the wholesale market and air temperatures in the range from 50 to $150 \mathrm{C}$ were made. It was found that the simple exponential equation described adequately the drying kinetic of these by-products. 
The following drying rate equation was obtained (L_opez, Iguaz, Esnoz, \& V_1rseda, 2000a) [12].

$$
R w=K(W-W e)
$$

$\mathbf{K}$ is the drying constant and it is related to the temperature of the drying air by:

$$
K=0.00719 \exp \left(-\frac{130.64}{T g}\right)
$$

The equilibrium moisture content (We) of the product was calculated by determining experimentally the equilibrium moisture isotherms at 25, 40,60 and 90. GAB model (Van Der Berg, 1984) was selected to predict We because it was the model that better fit to experimental data. The following expression was obtained (L_opez, Iguaz, Esnoz, \& V_irseda, 2000b) [12].

$$
W e=\frac{W_{m} C K a_{w}}{\left(1-K a_{w}\right)\left[1+(C-1) K a_{w}\right]}
$$

Where $\mathrm{Wm}, \mathrm{C}$ and $\mathrm{K}$ are parameters related to air temperature by the following expressions:

$$
\begin{aligned}
& W_{m}=0.0014254 \exp \left(\frac{1193.2}{T_{k}}\right) \\
& C=0.05923841 \exp \left(\frac{1072.5}{T_{k}}\right) \\
& K=1.00779919 \exp \left(-\frac{43.146}{T_{k}}\right)
\end{aligned}
$$

Where Tk is air absolute temperature $(\mathrm{K})$.

Substituting for (Rw) on model equations, and set the Dryer Plant parameters as next,

Table 1. Operating parameters of Plant Dryer

\begin{tabular}{|c|c|}
\hline Parameter & Value \\
\hline N_drum & $1.0 \mathrm{r} / \mathrm{m}$ \\
\hline Vg & $0.7 \mathrm{~m} / \mathrm{s}$ \\
\hline Vs & $4.78^{*} 10^{\wedge}-3 \mathrm{~m} / \mathrm{s}$ \\
\hline Fg & $0.12 \mathrm{~kg} / \mathrm{m}$ \\
\hline Fs & $8.77 \mathrm{~kg} / \mathrm{m}$ \\
\hline Tgin & $472 \mathrm{~K}$ \\
\hline Tg,out & $421 \mathrm{~K}$ \\
\hline Tsin & $293 \mathrm{~K}$ \\
\hline Ts,out & $360 \mathrm{~K}$ \\
\hline Xsin & $2.4 \%$ \\
\hline Xs,out & $0.01 \%$ \\
\hline Cg & $1.01 \mathrm{kj} / \mathrm{kg} \mathrm{K}$ \\
\hline Cs & $0.84 \mathrm{kj} / \mathrm{kg} \mathrm{K}$ \\
\hline Uv & $0.27 \mathrm{kj} / \mathrm{s} \mathrm{m}{ }^{\wedge} 3 \mathrm{~K}$ \\
\hline Vv & $0.19 \mathrm{~m}^{\wedge} 3 / \mathrm{m}$ \\
\hline lmd & $2261 \mathrm{kj} / \mathrm{kg}$ \\
\hline $\mathrm{L}$ & $\mathrm{m} \mathrm{3}$ \\
\hline Tk & $600 \mathrm{~K}$ \\
\hline \multicolumn{2}{|c|}{} \\
\hline
\end{tabular}

We can write down the system model in the canonical form of:

$\dot{\mathrm{X}}=\mathrm{AX}+\mathrm{Bu}, \quad \mathrm{Y}=\mathrm{CX}$

Where:

$\mathrm{X}=\left[\begin{array}{c}\mathrm{X}_{s, \text { out }} \\ \mathrm{T}_{s, \text { out }} \\ \mathrm{T}_{g, \text { out }}\end{array}\right], \quad\left[\begin{array}{c}\mathrm{T}_{g, \text { in }} \\ \mathrm{T}_{s, \text { in }} \\ \mathrm{X}_{s, \text { in }} \\ \mathrm{V}_{s} \\ \mathrm{~F}_{g} \\ \mathrm{~F}_{s}\end{array}\right]$
$\mathbf{A}=\left[\begin{array}{ccc}-0.0025933 & 0 & -1 e-009 \\ -2.6917 & -0.008557 & 0.006961 \\ -163.61 & 0.42327 & -0.65676\end{array}\right]$

$\mathbf{B}=$

$\left[\begin{array}{cccccc}0 & 0 & 0.0015933 & 0.0046667 & 0 & 0 \\ 0 & 0.0015933 & 0 & -22.333 & 0 & -0.0012185 \\ 0.23333 & 0 & 0 & 0 & -1105.3 & 15.198\end{array}\right]$

\section{$\mathbf{C}=\left[\begin{array}{lll}1 & 1 & 1\end{array}\right]$}

A sensitivity analysis shows that the main variable that needs to be manipulated in order to control the output moisture content of the solids is the drying air temperature which correlated to the fuel flow, and that the main disturbance variable is the input moisture content.

The feed flow can be used as an auxiliary variable to be manipulated, or else it can be a disturbance variable.

The existing operating conditions are simulated first to validate the model.

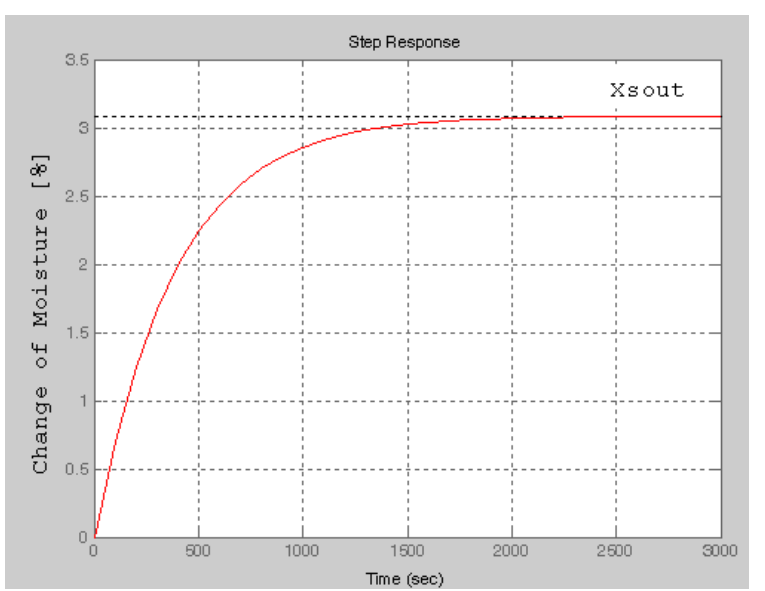

Fig 3: Simulated output responses for Xsout to a step change in the input moisture of solids from $2.4 \%$ to $4.4 \%$. 


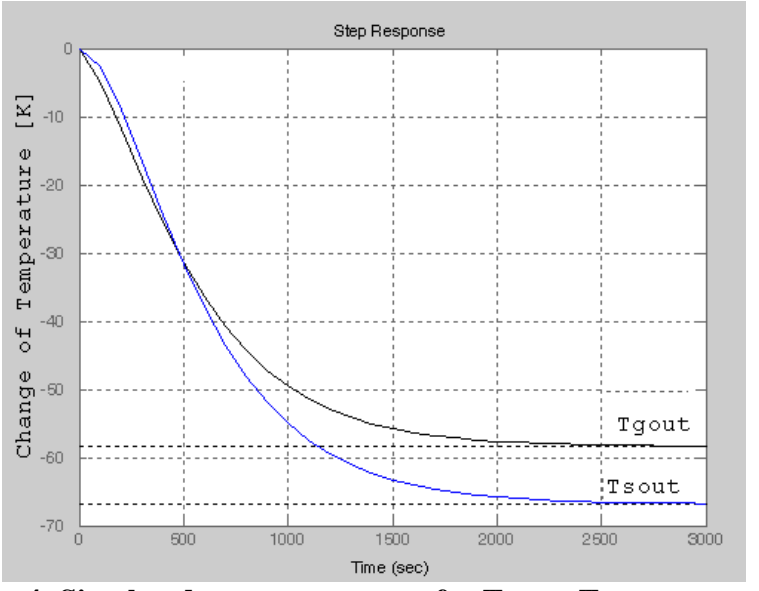

Fig 4: Simulated output responses for Tgout, Tsout to a step change in the input moisture of solids from $2.4 \%$ to $4.4 \%$.

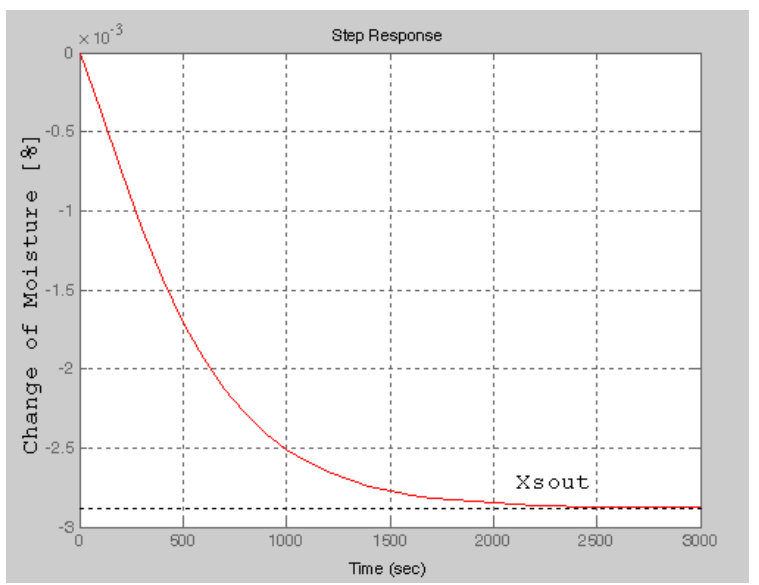

Fig 5: Simulated output responses for Xsout to a step change in the input temperature of the drying air from 463 $\mathrm{K}$ to $483 \mathrm{~K}$.

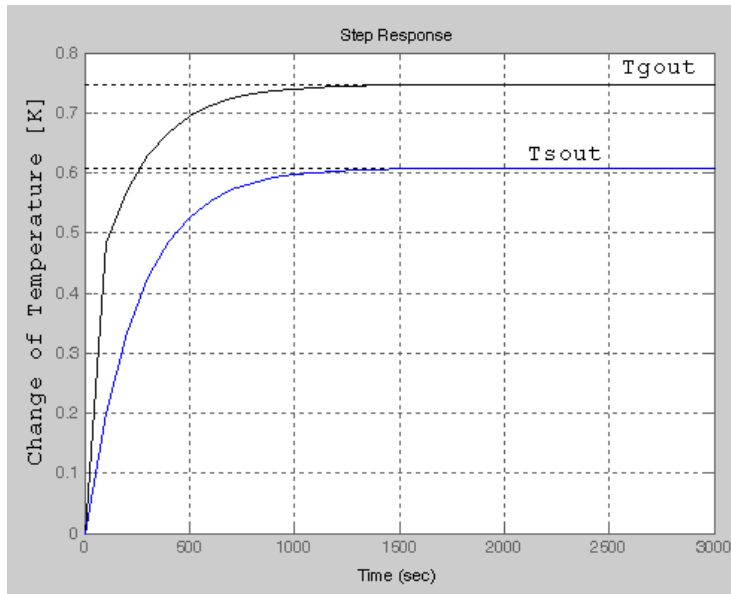

Fig 6: Simulated output responses for Tgout, Tsout to a step change in the input temperature of the drying air from 463 $K$ to $483 \mathrm{~K}$.

\section{FUZZY LOGIC CONTROLLER 3.1 Design procedure of the FLC plant rotary dryer:}

There are two methods of developing a self tuning fuzzy controller: one of them is based on neural networks whereas the other uses fuzzy logic. To keep the structure of the controller as simple as possible and to avoid heavy computation, a fuzzy logic supervisor controller based on meta rules is selected. Following the way proposed by Mudi and Pal the supervisor controller tunes the output scaling factors of the low-level controller using a gain updating factor [6]. Furthermore the inputs of the supervisor controller are selected the same as the ones of the low-level controller. This reduces the amount of memory required for the data base [7].

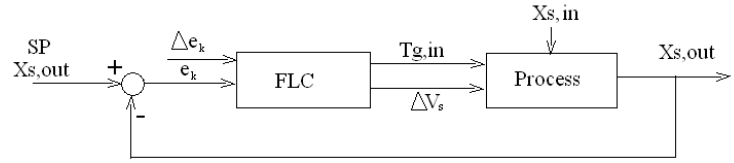

Fig 7: FLC Structure for Plant Rotary Dryer

The design procedure of the FLC for plant rotary dryer is as follows:

Step 1: System functional requirements. As the previous studies have proved the fuzzy logic controller applies to the control of a rotary dryer. The aim of the current FLC is to improve the performance of the existing fuzzy controllers by utilizing the operator's expert knowledge.

Step 2: Definition of system parameters. The main controlled variable is the output moisture of solids and the main manipulated variable is the input temperature of drying air, which correlates to the fuel flow. The velocity of solids which correlates to the rotational speed of the screw conveyor can be used as an auxiliary manipulated variable. The main disturbances are the variations in the input moisture of solids and the feed flow.

Step 3: Definition of system parameters in terms of fuzzy sets. The input variables for the FLC representing the contents of rule antecedents are as follows:

- $\quad$ Error in the output moisture of solids (input 1)

- Change in the error of the output moisture of solids (input 2);

- The controller output representing the contents of the rule consequent is as follows:

- Gain updating factor (output 1); for input drying air temperature correlated to fuel flow.

- Gain updating factor (output 2); for change in velocity of solids inside drum correlated to feed speed.

Where: $e_{k}=\mathrm{X}_{s, \text { out }}^{\mathrm{SP}}-\mathrm{X}_{s, \text { out }}$ and $d e_{k}=\mathrm{S}_{\mathrm{i}=1}^{\mathrm{k}-1} e_{i}$

Step 4: Formulation of control rules. The control rule based on the analysis of the controlled response and on the experience is of the form:

IF input 1 is $\{\mathrm{I} 1 \mathrm{j}\}$ AND input 2 is $\{\mathrm{I} 2 \mathrm{j}\}$ THEN output is zero, small, medium, big, very big $\}$, where $\mathrm{I} 1 \mathrm{j}$ and $\mathrm{I} 2 \mathrm{j}$ are fuzzy sets defined in the low level controller data base.

The number of rules depends on the number of labels for the input linguistic variables chosen for the supervisor controller.

In this application the fuzzification is made using the Min-Max algorithm. The inference engine or rule firing is based in the one-level forward data-driven inference, which is the generalization of the modus ponens rule of inference [6].

Step 5: Selection a method of defuzzification. The centre of area method (COA) is used to transform the output of the fired rules into the crisp value. 
Step 6: Simulation tests. The controller has been implemented in Matlab®, using Simulink and Fuzzy Logic Toolbox. Simulations have been carried out using the the direct FLC as controllers and making step changes in the main disturbance variable i.e. the input moisture of solids.

See appendix 1 for the Trapezoidal membership functions.

\begin{tabular}{|c|c|c|c|c|c|c|}
\hline \multicolumn{2}{|l|}{$\begin{array}{l}\text { Error in output } \\
\text { Moisture of solids }\end{array}$} & \multicolumn{2}{|c|}{ Change in error } & \multicolumn{2}{|l|}{$\begin{array}{l}\text { Gain updating } \\
\text { factor Tg,in }\end{array}$} & \multirow{2}{*}{$\begin{array}{l}\begin{array}{l}\text { Gain updating } \\
\text { factor Vs }\end{array} \\
\text { small }\end{array}$} \\
\hline 1 IF very negative & AND & negative & THEN & very big & AND & \\
\hline 2 IF negative & AND & zero & THEN & medium & AND & big \\
\hline 3 IF small negative & AND & positive & THEN & small & AND & very big \\
\hline 4 IF very small negative & AND & negative & THEN & big & AND & medium \\
\hline 5 IF zero & AND & zero & THEN & zero & AND & zero \\
\hline 6 IF very small positive & AND & positive & THEN & big & AND & small \\
\hline 7 IF small positive & AND & negative & THEN & small & AND & very big \\
\hline 8 IF positive & AND & zero & THEN & medium & AND & big \\
\hline 9 IF very positive & AND & positive & THEN & very big & AND & small \\
\hline 10 IF very negative & AND & zero & THEN & medium & AND & big \\
\hline 11 IF negative & AND & negative & THEN & big & AND & medium \\
\hline 12 IF small negative & AND & negative & THEN & big & AND & medium \\
\hline 13 IF very small negative & AND & zero & THEN & medium & AND & big \\
\hline $14 \mathrm{IF}$ zero & AND & negative & THEN & big & AND & medium \\
\hline 15 IF very small positive & AND & negative & THEN & small & AND & very big \\
\hline $16 \mathrm{IF}$ small positive & AND & zero & THEN & medium & AND & big \\
\hline 17 IF positive & AND & negative & THEN & small & AND & very big \\
\hline 18 IF very positive & AND & negative & THEN & small & AND & very big \\
\hline 19 IF very negative & AND & positive & THEN & small & AND & very big \\
\hline 20 IF negative & AND & positive & THEN & small & AND & very big \\
\hline 21 IF small negative & AND & zero & THEN & medium & AND & big \\
\hline 22 IF very small negative & AND & positive & THEN & small & AND & very big \\
\hline $23 \mathrm{IF}$ zero & AND & positive & THEN & big & AND & medium \\
\hline 24 IF very small positive & AND & zero & THEN & medium & AND & big \\
\hline $25 \mathrm{IF}$ small positive & AND & positive & THEN & big & AND & medium \\
\hline 26 IF positive & AND & positive & THEN & big & AND & medium \\
\hline 27 IF very positive & AND & zero & THEN & medium & AND & big \\
\hline
\end{tabular}

\subsection{Design of the direct FLC}

The structure of the direct FLC is presented as the trapezoidal membership functions have been selected to the different linguistic variables and their labels of the direct FLC to keep the controller simple.

The membership functions of the output (gain updating factor) of the FLC have been chosen as in the previous case. The different linguistic variables and their labels are showed in the next Table.
Variable

Label

Error in the output moisture of solids:

Very negative

Negative

Small negative

Very small negative

Zero

Very small positive

Positive

Very positive

Variable

Label

Change in the error of the output moisture of solids:

Negative

Zero

Positive

Variable

Label

Gain updating factor for (Tgin, Vs):

Small

Medium

Zero

Big

Very big 


\subsection{Applying Fuzzy Logic Controller on Plant Dryer.}
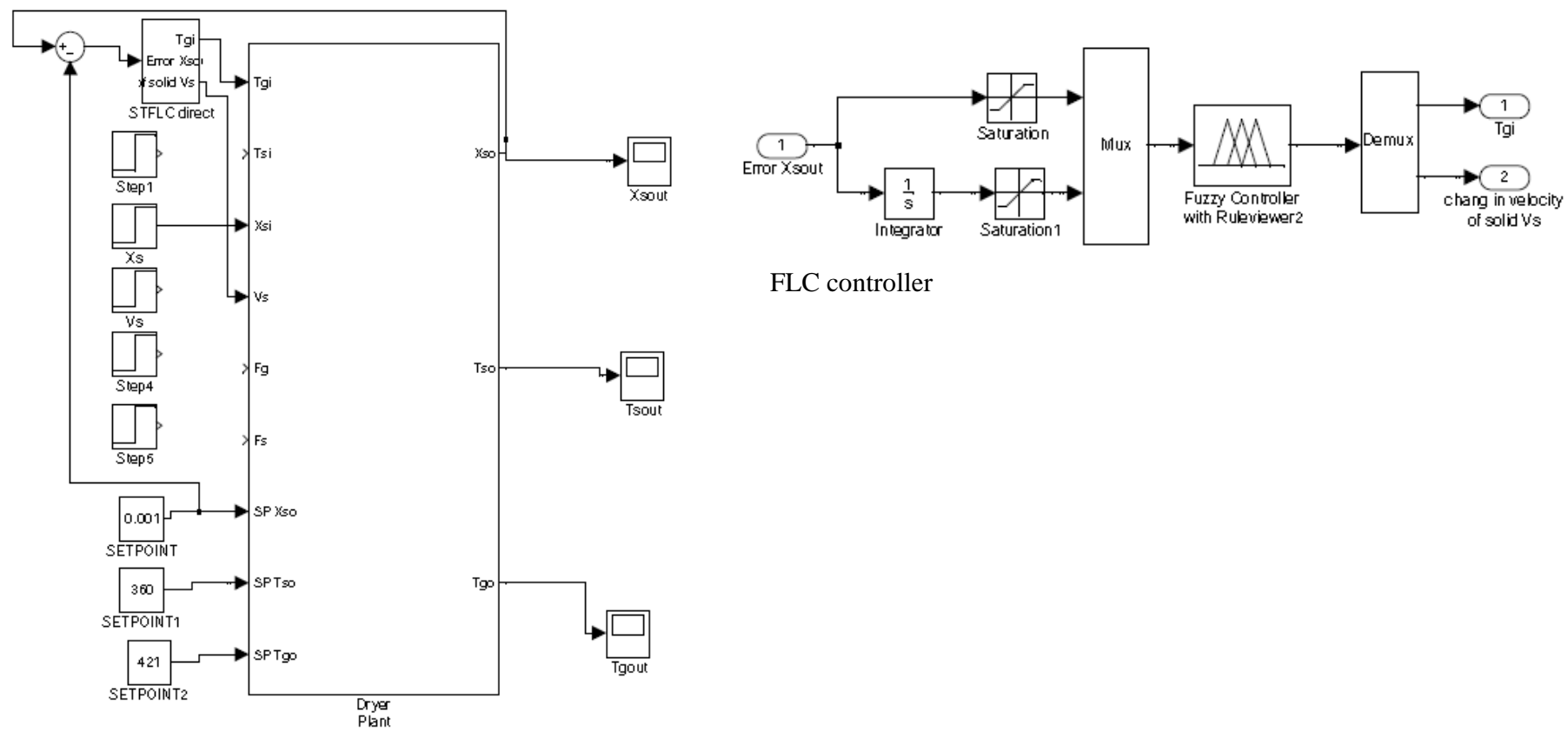

FLC controller

Fig 8: Simulink block diagram for dryer plant connected to the FLC controller

\section{NEURO-FUZZY CONTROLLER}

Recently, the combination of neural networks and fuzzy logic is getting more attention. Neural networks bring to this union the ability to learn, but also require an excessive number of iterations for training of complex systems. Fuzzy logic offers system models based on membership functions and a rule base, but require an explicit stating of the IF/THEN rules.

Figure 8 illustrates the system architecture for the described combination of neural networks and fuzzy logic. By replacing the rule base of a fuzzy system with a trainable neural network, complex input-output relationships can be achieved which cannot be easily specified by fuzzification and defuzzification stages augmenting a neural network, significant improvements in the training time, in the ability to generalize, and in the ability to find minimizing weights can be realized. Furthermore, the fuzzy membership functions give the designer more control over the neural network inputs and outputs.

Neuro-fuzzy controllers constitute a class of hybrid Soft Controllers that fuse fuzzy logic and artificial neural networks. Though the principles of fuzzy logic and artificial neural networks are very different, the two techniques have important common features: fuzzy logic aims at repro-ducing the mechanism of human cognitive faculty while neural networks attempt to emulate the human brain at the physiological level.

In fuzzy controllers linguistic rules embody the knowledge on how to control a physical plant.

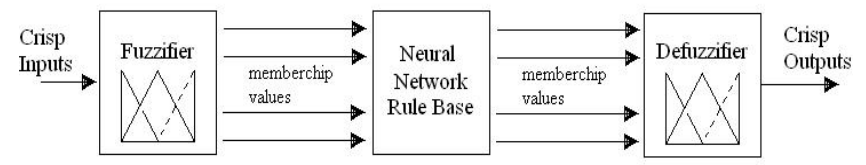

Fig 9: A fuzzy system with neural network rule base

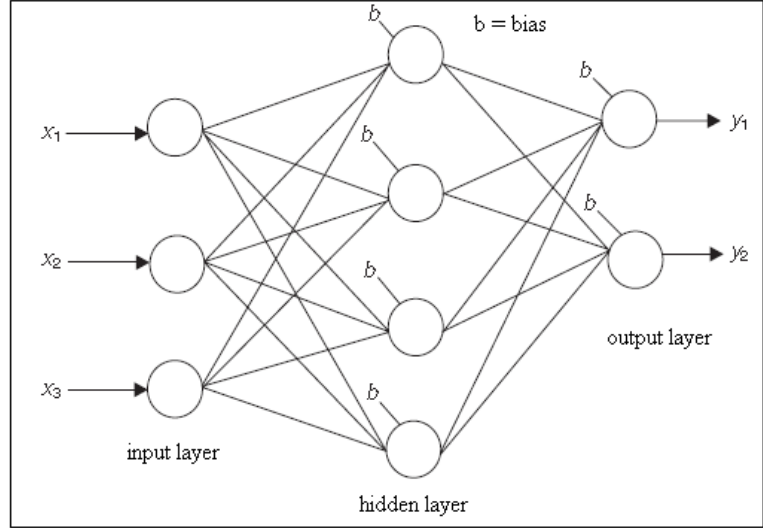

Fig 10: Multi-layered neural network

In a neural controller this knowledge is embedded in the structure and the synaptic weights of the network. Feed-forward processing in ANNs is analogous to the inference engine in fuzzy logic. Fuzzy controllers use fuzzy compositional rules to arrive at their decisions, require fuzzification of the input variables and defuzzification of the composite fuzzy set of the output in order to obtain a crisp output from the controller. In contrast, neural controllers use simple arithmetic techniques, operating directly in the physical world. In both cases, current data from the physical plant being controlled is stored in a real-time database and then processed by an appropriate algorithm. Only in the manner with which the two techniques arrive at this control action do they differ radically. The ability to generalize, i.e., to extrapolate when faced with a new situation, is a feature common to both.

Evolving from very different origins, fuzzy and neural controllers developed independently by researchers with very different back-grounds and very different objectives.

\subsection{Neuro-Fuzzy Controller Architectures}

It is logical to examine the fusion of fuzzy logic and ANNs with a view to developing hybrid neuro-fuzzy controllers that possesses the best at-tributes of both techniques in the hope that 
this will lead to a superior class of intelligent controller [9]. In principle it should be possible to "neu-ralize" a fuzzy controller or "fuzzify" a neural controller. It is useful, therefore, to state the principal characteristics of hybrid neuro-fuzzy controllers [11]:

- They possess an architecture derived from both techniques,

- They have elements of both fuzzy and neural controllers, each of which performs a separate task and

- their design methodology is a combination of the two techniques.

A number of neuro-fuzzy controller architectures have been proposed, each with features that make them suitable for specific applications. Considering fuzzy and neural elements as distinct entities, it is possible to construct a controller structured in layers, some of which are implemented with neural elements and others with fuzzy elements. A fuzzy element can, for instance, act as supervisor to a neural element that controls some conventional industrial three-term controller.

The following characteristics of ANNs are useful in implementing fuzzy controllers: they

- use a distributed representation of knowledge,

- are macroscopic estimators,

- are fault-tolerant and

- can deal with uncertainty and vagueness.

\subsection{Designing Neuro-Fuzzy Controller for the Rotary Dryer}

Sample training data for Adaptive Neuro Fuzzy controller:

Loading training data:

Function used to generate training data

For ANFIS:

$\mathrm{Y}=(\sin (2 * \mathrm{x}) / \exp (\mathrm{x} / 5)) *$ factor;

For Input of controller:

MF s type Gauss Member function

Number of MF is 12

For Output of controller:

MF type Linear

Neuro Fuzzy after Training

Comparing with original data

Using Error tolerance 0

And Hybrid Method for training

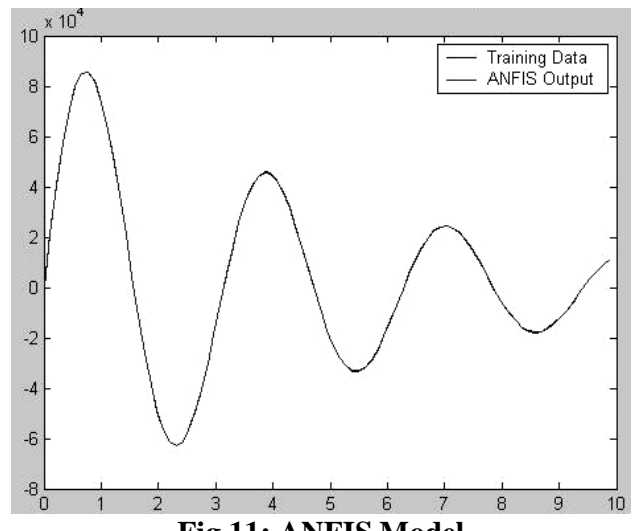

Fig 11: ANFIS Model

(Training data versus ANFIS Output)

\section{SIMULATION RESULTS}

Upper bound of disturbance applying on Xs,in (input moisture content of solids) is $2.00 \%$, and the lower bound is $5.00 \%$. The normal value of $\mathrm{Xsin}$ is $2.4 \%$ (operating point), Testing the system with disturbance from $6.66 \%$ to $80 \%$ to see the response of Plant Dryer with difference types of controllers , (Using Matlab and simulink).

\subsection{Summarizing the results}

Table 2. Response of the Plant Dryer when connected to difference types of controllers (testing the system with disturbance from $6.66 \%$ to $80 \%$ ).

\begin{tabular}{|c|c|c|c|c|}
\hline & $\begin{array}{c}\text { Rising } \\
\text { Time } \\
\text { /Min }\end{array}$ & $\begin{array}{c}\text { Settling } \\
\text { Time } \\
\text { /Min }\end{array}$ & $\begin{array}{c}\text { Max. } \\
\text { Overshoot } \%\end{array}$ & $\begin{array}{c}\text { Steady } \\
\text { State Error } \\
\%\end{array}$ \\
\hline PID & 4.00 & 19 & 0.2500 & $\sim 0$ \\
\hline Fuzzy & 3.00 & 17 & 0.0130 & 0.00011 \\
\hline $\begin{array}{l}\text { Neuro } \\
\text { Fuzzy }\end{array}$ & 1.00 & 12 & 0.0090 & 0.00005 \\
\hline
\end{tabular}

\subsection{Applying the Neuro-Fuzzy controller}

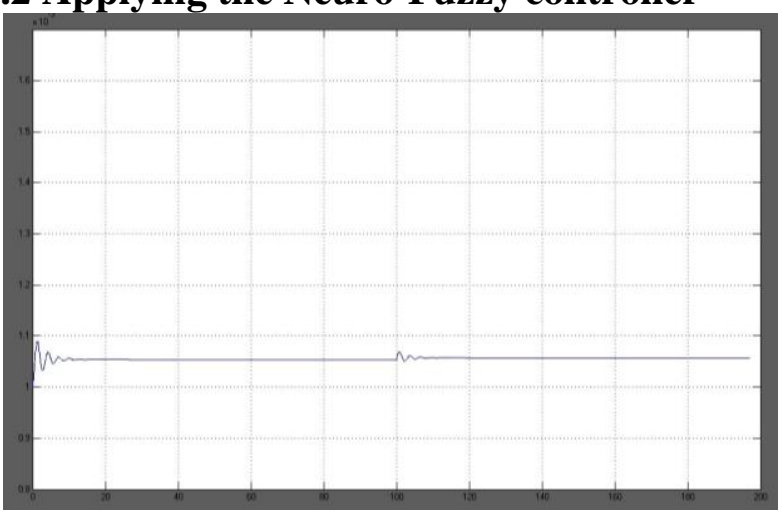

Fig 12: Output response of Dryer Plant connected to the Neuro-Fuzzy controller Xs, out for Step change of Xs,in (Main disturbance) from $2.4 \%$ to $4.4 \%$ at time of $100 \mathrm{sec}$.

\subsection{Applying the FLC controller}

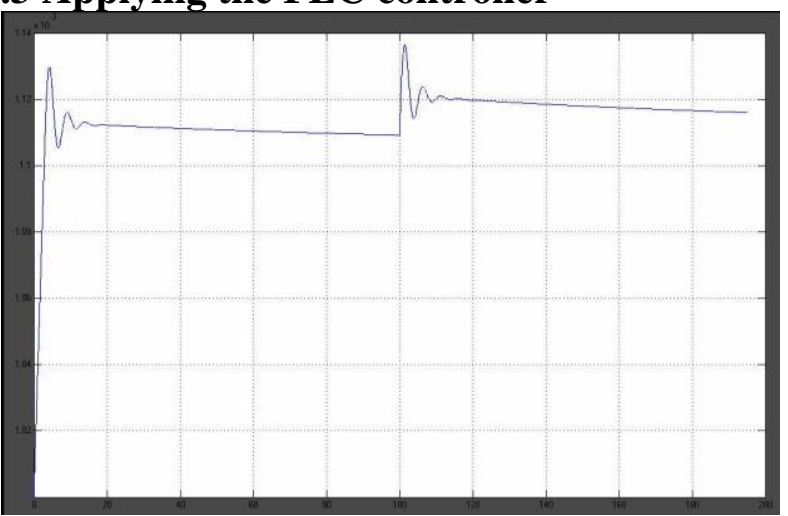

Fig 13: Output response of Dryer Plant connected to the FLC controller Xs, out for Step change of Xs,in (Main disturbance) from $2.4 \%$ to $4.4 \%$ at time of $100 \mathrm{sec}$.

\subsection{Applying PID controller}

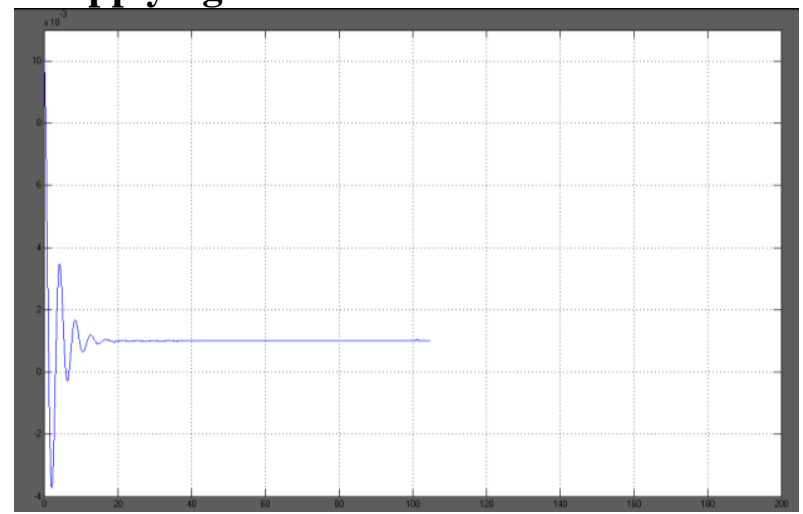

Fig 14: Output response of Dryer Plant connected to the direct PID controller. 


\section{CONCLUSION}

In this paper we make combination between two dynamic models of dryer plant using the drying rate equation which cause the non-linearity of the model as in Iguaz [12], with the model in Yliniemi [4]. Then three different control systems are presented to apply and validate some modern adaptive control techniques, Direct PID controller, Fuzzy logic controller (FLC) and NeuroFuzzy controller, in order to control the Rotary Dryer plant process, then see the behavior of the plant specially for the main controlled variable (output Moisture content of solids), after applying the different controllers, comparing all results with the behavior of the plant when applying the direct Fuzzy logic controller also with the traditional PID controller, After reviewing the operation of the plant, The comparison between these different control systems was made by simulations when a step change in the input moisture of solids occurs. The simulation parameters are based on the pilot plant dryer Yliniemi [4]. The control results have been compared with the results achieved by the traditional PID-controller.

Simulations are presented and shows that Neuro-Fuzzy control are better and more versatile compared with the conventional approach using PID controller, and with direct fuzzy controller designed, when applying all this combinations on the dryer plant with the same step change in the main disturbance of input moisture content of solids.

Complete simulations on four versions of controllers were done namely:

a- Direct Fuzzy Logic Controller.

b- Neuro-Fuzzy Controller.

c- Conventional PID controller.

It can be concluded that with the produced combined dynamic model of the plant dryer the proposed Neuro-Fuzzy controller yielded a better dynamic performance than the other two controllers, in terms of Rising Time, Settling Time, Maximum Overshoot and Steady-State Error, and this is a limited problem concerned only with the case study verified by simulation.

\section{REFERENCES}

[1] L. Pirrello 1, L. Yliniemi 2, K. Leiviskä2 and M. Galluzzo 1 2002 Self-Tuning fuzzy control of a rotary dryer .

[2] Leena Yliniemi, 2001 Adaptive Fuzzy Control of a Rotary Dryer, In: Industrial Applications of Soft Computing (K. Leiviska, Ed.). Studies in Fuzziness and Soft Computing, Vol. 71, Springer Verlag.

[3] Carmen Jover, Carlos F. Alastruey 2004 Multivariable control for an industrial rotary dryerDepartment of Automatic Control and Computing Systems, Public University of Navarra, 31006 Pamplona, Spain.

[4] Yliniemi, Leena, 1999 Advanced control of a rotary dryer Department of Process Engineering, University of Oulu, FIN-90570 Oulu.

[5] Luca Cammarata and Leena Yliniemi 1999 Development of a Self-Tuning Fuzzy Logic Controller (STFLC) for a Rotary Dryer.

[6] Leonardo Pirrello, Leena Yliniemi, Kauko Leiviskä 2001 Development of a Fuzzy Logic Controller for a Rotary Dryer with Self-Tuning of Scaling Factor.

[7] Davide Fratantonio, Leena Yliniemi and Kauko Leiviskä 2001 Fuzzy modelling for a rotary dryer.

[8] Harshdeep Singh Ahluwalia 2002 Modeling and Optimization of a Rotary Dryer in a Manufacturing Plant.

[9] Tsourveloudis, N.C., Kiralakis, L. , 2005 Rotary Drying of Olive Stones: Fuzzy Modeling and Control, WSEAS Transactions on Systems 4 (12), pp. 2361-2368
[10] M.G. Ortega_, F. Castan o, M. Vargas, F.R. Rubio 2005 Multivariable robust control of a rotary dryer: Analysis and designDepto. Ingenieri'a de Sistemas y Automa'tica, Universidad de Sevilla, Escuela Superior de Ingenieros, Camino de los Descubrimientos sn, 41092 Sevilla, Spain Received 27 April (2005).

[11] Francisco A. Cubillos a, Pedro I. Alvarez \% Jose C. Pinto b Enrique L. Lima u 1995 Hybrid-neural modeling for particulate solid drying processes

[12] Iguaz , A. Esnoz , G. Mart_inez , A. L_opez , P. V_rrseda 2003 Mathematical modelling and simulation for the drying of vegetable wholesale by-products in a rotary dryer Journal of Food Engineering 59 (2003) 151-160

[13] J. R. Pkez-Correa,F. Cubillos, E. Zavala, C. Shene and P. I. Alvarez Dynamic simulation and control of direct rotary dryers.

\section{APPENDIX 1}

Trapezoidal membership functions:
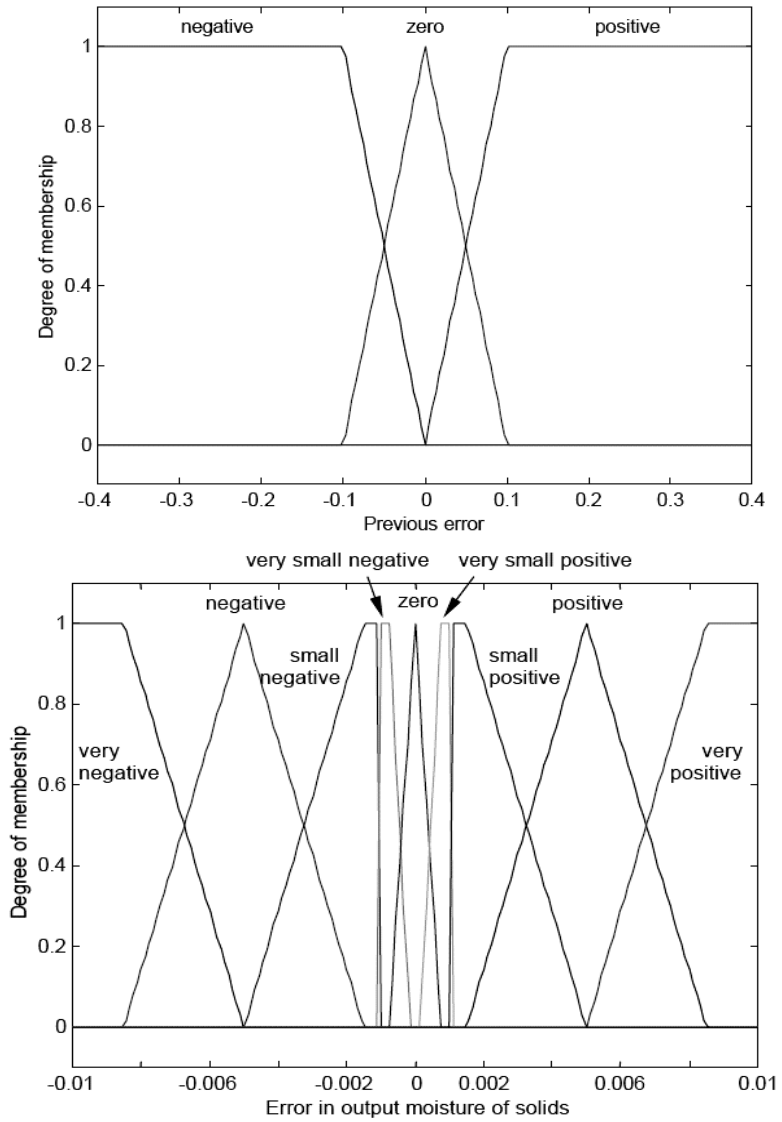

Trapezoidal membership functions of the input variables the direct FLC.

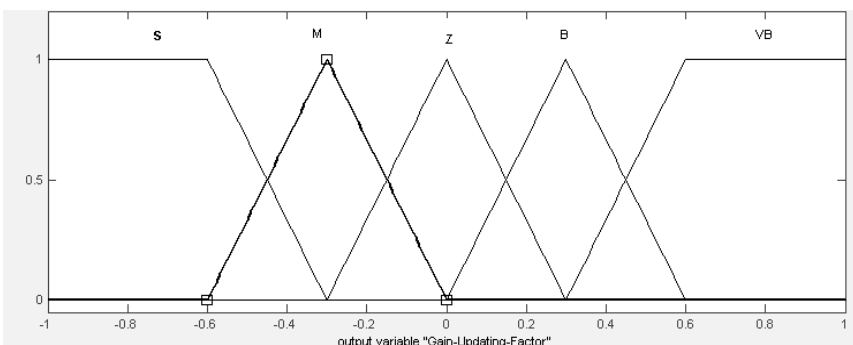

Trapezoidal membership functions of the output variables of the direct FLC. 